\title{
ECOLOGIE DES CÉRATOPOGONIDÉS DE LA PLAINE D'ALSACE
}

\section{III - Cycle évolutif des Culicoides (Diptères, Cératopogonidés) d'un gîte fluviatile.}

\author{
J.-P. RIEB ET M. KREMER*
}

(Collaboration technique : J.-c. DELECoLLE)

RÉSUMÉ. Dans l'Est de la France, Culicoides clastrieri, C. odibilis, C. cubitalis, C. subfascipennis et $C$. musilator présentent 2 générations par an; une génération de printemps et une génération d'été. Une partie de la population de l'été subit une estivo-hibernation qui est levée vers le mois de décembre. La possibilité d'une diapause hivernale est envisagée.

\section{Ecology of the Ceratopogonids of the Alsace plain. III. Life-cycle of the Culicoides (Diptera, Ceratopogonidae) from a fluvial breeding-place.}

SUMMARY. In the East of France, Culicoides clastrieri, $C$. odibilis, C. cubitalis, C. subfascipennis and $C$. musilator have 2 generations per year; a spring generation and a summer generation. A part of the summer population undergoes a aestivo-hibernation which is terminated around december. The possibility of a winter diapausa is evocated.

Dans un travail précédent, nous avons étudié la répartition spatiale des larves de Culicoides sur le gite fluviatile d'Ichtratzheim (1) en plaine d'Alsace (France), ainsi que les facteurs caractérisant ce gite (Rieb et Kremer 1980).

La dynamique de population des différentes espèces est étudiée sur les mêmes prélèvements que ceux utilisés pour l'étude de la répartition spatiale. Chaque semaine, nous réalisons une série de 9 prélèvements de boues sur un transect perpendiculaire à la rive comportant 9 stations équidistantes qui sont, de la berge vers la rivière $: \alpha, \mathrm{A}$, B, C, D, E, F, G, H. Ces boues, maintenues en laboratoire, donnent éclosion aux imagos qui sont capturés tous les 2 ou 3 jours.

Nous réalisons, pour chaque espèce, un tableau sur lequel sont représentées sous forme d'histogrammes, les éclosions de chaque série de prélèvements en fonction du

* Laboratoire de Parasitologie et de Pathologie tropicale, Faculté de Médecine, 3, rue Koeberlé, F 67000 Strasbourg.

I. Coordonnées : $4^{\circ} 28^{\prime} 30^{\prime \prime}$ Nord, $7^{\circ} 4 \mathrm{I}^{\prime} 20^{\prime \prime}$ Est de Greenwich.

Accepté le zo janvier I98I. 
temps passé au laboratoire. La somme des éclosions des prélèvements $\alpha$ à $H$ d'une même série est faite tous les 3 jours ; la valeur numérique correspondante est représentée sur l'histogramme en ordonnée dégressive. L'histogramme complet de chaque série de prélèvements est donc l'image de la composition de la population larvaire présente dans la boue au jour du prélèvement, en nombre et en âge. La succession des histogrammes en fonction des dates de prélèvements (ordonnées) indique l'importance et la durée d'une population larvaire et permet de reconnaître les générations de larves.

Le temps de latence, c'est-à-dire le temps écoulé entre la date du prélèvement et la date d'éclosion de l'imago est fonction de l'âge ou de l'état physiologique de la larve au moment du prélèvement : s'il est de 24 à 48 heures, l'individu prélevé était à l'état de nymphe ou de larve au stade IV prête à nymphoser. Plus ce temps de latence est long, plus la larve était jeune. S'il est plus long que la durée de développement normale des individus les plus jeunes d'une génération, la larve était en état de diapause.

Parallèlement à cette étude en laboratoire, nous avons contrôlé sur le terrain les émergences des adultes grâce à des pièges d'émergence. Les résultats sont indiqués à gauche des tableaux : la chronologie des dates est en ordonnées ; l'importance numérique des émergences est représentée en absisses selon la même échelle dégressive que les ordonnées des histogrammes de populations larvaires.

Nous avons réalisé les tableaux d'histogrammes pour les 5 espèces de Culicoides numériquement les plus importantes : $C$. clastrieri, $C$. odibilis, $C$. cubitalis, $C$. subfascipennis et C. musilator (fig. 1 à 5 ).

Les résultats montrent que les 5 espèces ont le même cycle évolutif. Il y a 2 générations par an ; une génération de printemps issue de larves ayant passé l'hiver (et dont nous avons contrôlé la présence en grand nombre par la technique de flottation), et une génération d'été. La population de cette $2^{\mathrm{e}}$ génération est complexe, car une partie des larves évolue normalement en donnant éclosion à des imagos pendant une période allant jusqu'à 2 mois à 2 mois et demi de la date de prélèvement, alors que d'autres larves se développent lentement et présentent une éclosion différée, pouvant avoir au laboratoire un retard de 2 à 15 mois par rapport à la génération d'été normale.

\section{A - Cycle évolutif}

\section{$1-$ C. clastrieri (fig. 1)}

Cette espèce, qui est la plus nombreuse sur le gite, présente au printemps 1977 une génération d'importance numérique moyenne (167 individus dans le prélèvement du 20.5.77). Ces larves, qui ont passé l'hiver, sont présentes dans les prélèvements jusqu'au 10 juin. Les émergences apparaissent sur le terrain le 3 juin et durent jusqu'au 21 juin, date limite de la génération larvaire de printemps. La génération d'été révèle des larves du 17 juin au 2 septembre. Les émergences sont trop faibles pour 
préciser la date de vol des imagos d'été : 1 individu à chaque fois les 8 juillet, 16 août et 2 septembre!

Les larves à éclosion différée apparaissent en même temps que les larves d'été à partir du 17 juin 1977, et sont présentes jusqu'au 18 novembre. Les prélèvements d'hiver et de printemps n'en révèlent plus (à l'exception de 2 individus le 14 février 1978). Au laboratoire, les éclosions différées les plus tardives ont lieu jusqu'à 477 jours après la date de prélèvement.

Les larves de la génération de printemps 1978 sont présentes à partir du 9 au 16 septembre 1977. La population reste très importante pendant l'hiver et donne au printemps 1978 une éclosion massive (1277 individus le 25.4.78).

La comparaison entre les générations de printemps de 1977 et de 1978 sur des périodes de prélèvement identiques, montre que la génération de printemps 1978 est numériquement 11 fois plus importante que celle de 1977. Cette génération de printemps 1978 est également de plus grande durée : les larves sont présentes jusqu'au 25 juillet, c'est-à-dire 45 jours de plus qu'en 1977 ! Les émergences de printemps, relativement importantes, existent du 16 mai au 18 juillet.

Les larves de la génération d'été et les larves à éclosion différée apparaissent en même temps le 13 juin. Les émergences d'été existent du 25 juillet au 8 août.

On constate qu'en 1977 les deux générations se succèdent avec un très faible recouvrement entre le 17 et le 21 juin. Par contre en 1978, du fait de la grande durée de la génération de printemps, il y a chevauchement des deux générations entre le 13 juin et le 25 juillet.

Dans une série de prélèvements de même date, les larves faisant partie d'une même génération donnent au laboratoire des éclosions d'adultes réparties sur une période d'environ 80 jours. Par exemple, les éclosions de la série de prélèvements du 1 er juillet 1977, s'échelonnent de 5 à 77 jours après la date du prélèvement. Cette répartition des éclosions des individus de la génération d'été est plus ou moins symétrique. Cet échelonnement des éclosions est dû, d'une part aux variations individuelles de vitesse de croissance des larves, et d'autre part au fait que cette population de larves est issue de pontes déposées par plusieurs femelles, a des dates différentes. Par contre, dans les éclosions de printemps (histogrammes de mai 1978), la répartition est dissymétrique; le pic est déplacé vers la gauche, c'est-à-dire vers les éclosions précoces. Ces individus ont été pondus en été 1977 - certes à des dates différentes mais ils ont vécu pendant 9 mois en vie ralentie sous la contrainte des conditions climatiques : leur différence d'âge au moment du retour aux conditions favorables à l'éclosion est donc négligeable. Ainsi la plupart des individus éclosent de façon groupée dès que la température est assez élevée. Il reste cependant des éclosions échelonnées qui sont dûes aux différences de vitesse de croissance individuelle.

- Durée d'un cycle de développement individuel dans la nature :

Il est possible d'estimer la durée d'un cycle de développement de la génération d'été sur les individus les plus précoces de cette génération. Il s'agit du temps séparant la ponte de l'œuf par une femelle éclose au printemps et issue d'une larve hivernante, et l'apparition d'une femelle estivale. 
Considérons le printemps 1977 où les générations sont clairement séparées. Les prélèvements du 17 juin contiennent les premières larves de la génération d'été qui, au laboratoire, donnent naissance au premier adulte après 22 jours. Ces individus ont été pondus entre le 10 et le 17 juin puisque les prélèvements du 10 juin ne contiennent pas encore de larves d'été. Ce cycle a donc une durée comprise entre $22+0=22$ jours et $22+7=29$ jours.

On peut cependant faire remarquer que les 22 jours passés au laboratoire, l'ont été à une température éventuellement supérieure à celle du terrain, et que le développement a ainsi été accéléré. Le calcul peut se faire d'une autre façon en considérant le temps écoulé entre le moment probable de la ponte (10 au 17 juin) et la date de la première série de prélèvements donnant des adultes très peu de temps après son transport au laboratoire, et contenant donc des larves prêtes à nymphoser. La série prélevée le 1 er juillet est dans ce cas; la première éclosion d'adulte a lieu 5 jours après cette date. La durée du cycle de développement serait alors comprise entre $14+5=$ 19 jours et $21+5=26$ jours.

La durée d'un cycle de développement de la génération de printemps est beaucoup plus grande. Cette génération est issue de pontes de l'été précédent : les dernières émergences ont lieu début septembre en 1977 ; les pontes déposées en août ou début septembre donnent des larves qui mènent une vie ralentie en hiver. Les premières émergences apparaissent le 16 mai 1978 et se continuent jusqu'au 25 juillet 1978 . Ce cycle de développement dure donc de 9 à 11 mois.

\section{$2-$ C. odibilis (fig. 2)}

La génération de printemps 1977 est relativement faible (38 individus le 29.4.77) ; les larves sont présentes dans les prélèvements jusqu'au 10 juin. Émergences du 13 mai au 17 juin. La génération d'été est assez importante (267 individus le 22.7.77). Ell débute entre le 3 et le 10 juin et se termine entre le 2 et le 9 septembre. La répartition des éclosions est légèrement dissymétrique, avec un décalage du pic vers la gauche. Émergences du 12 juillet au 9 septembre. Larves à éclosion différée présentes à partir du 17 juin, mais surtout importantes du 5 août au 28 octobre. Au laboratoire, les éclosions différées les plus tardives ont lieu jusqu'à 387 jours après la date de prélèvement.

Larves de la génération de printemps 1978 présentes à partir du 9 au 16 septembre 1977. L'éclosion de printemps 1978 est massive (1016 individus le 25.4.78). Elle est numériquement 17 fois plus importante que celle de 1977. Les larves sont présentes dans les prélèvements jusqu'au 4 juillet 1978 (24 jours de plus qu'en 1977). Les éclosions ont une répartition dissymétrique, avec un pic déplacé vers la gauche, comme chez $C$. clastrieri. Les émergences de printemps existent du 9 mai au 11 juillet. Les larves de la génération d'été apparaissent le 30 mai et les larves à éclosion différée sont présentes à partir du 6 juin. Émergences du 25 juillet au 5 septembre. Les générations de printemps et d'été 1978 se chevauchent entre le 30 mai et le 11 juillet.

La durée des cycles de développement est voisine de celle de $C$. clastrieri, mais les générations de $C$. odibilis sont en général plus précoces (jusqu'à 15 ou 20 jours). 


\section{$3-$ C. cubitalis (fig. 3).}

La génération de printemps 1977 est assez importante (114 individus le 6.5.77), les larves sont présentes dans les prélèvements jusqu'au 8 juillet. Émergences du 31 mai au 21 juin. Génération de larves d'été à partir du 17 juin. Les effectifs deviennent faibles et les larves disparaissent vers le début de septembre. Émergences du 8 au 19 juillet. Les larves à éclosion différée apparaissent le 1er juillet. Elles sont numériquement importantes et sont présentes jusqu'au 10 novembre. Au laboratoire, les éclosions différées les plus tardives ont lieu jusqu'à 496 jours après la date de prélèvement.

Les larves de la génération de printemps 1978 apparaissent vers le 23 septembre 1977. L'éclosion de printemps 1978 est à peine plus importante que celle de 1977. Les larves sont présentes dans les prélèvements jusqu'au 25 juillet. Émergences à partir du 16 mai, et probablement jusque vers le 25 juillet (les émergences des deux générations se suivent apparemment sans discontinuité). Apparition des larves de la génération d'été le 11 juillet, mais probablement présentes plus tôt car les premières larves à éclosion différée existent déjà le 4 juillet. Les deux générations se chevauchent et sont mal délimitées. Émergences probables vers le 25 juillet et présentes jusqu'au 26 septembre.

\section{$4-$ C. subfascipennis (fig. 4)}

La génération de printemps 1977 est assez importante (123 individus le 29.4.77), les larves sont présentes dans les prélèvements jusqu'au 10 juin. Émergences du 24 mai au 14 juin. Puis l'espèce devient extrêmement rare sur le gite. Seuls quelques individus isolés ponctuent la génération d'été entre le 8 juillet et le 5 août. Aucune émergence d'été. Des larves à éclosion différée sont présentes entre le 5 août et le 21 octobre. Au laboratoire, les éclosions différées les plus tardives ont lieu jusqu'à 344 jours après la date de prélèvement.

Les larves de la génération de printemps 1978, très peu nombreuses, apparaissent à partir du 9 septembre 1977. Elles sont présentes dans les prélèvements jusqu'au 16 mai 1978, et existent sans doute plus tardivement encore, puisque un seul exemplaire représente l'émergence de printemps le 6 juin 1978. La génération d'été 1978 est pratiquement inexistante : 2 larves le 4.7.78, pas d'émergences et pas de larves à éclosion différée !

\section{$5-$ C. musilator (fig. 5)}

Cette espèce présente une évolution dans le temps inverse de celle de $C$. subfascipennis. La génération de printemps 1977 est extrêmement faible; de rares larves sont présentes dans les prélèvements jusqu'au 10 juin. Pas d'émergences. Larves de génération d'été présentes du 24 juin au 26 août. Une émergence le 12 juillet et une le 2 septembre. Larves à éclosion différée du 5 août au 28 octobre. Au laboratoire, les éclosions différées les plus tardives ont lieu jusqu'à 384 jours après la date de prélèvement. 
Les larves de la génération de printemps 1978 apparaissent le 23 septembre 1977. L'éclosion de printemps 1978 est nettement plus importante que celle de 1977 (46 individus le 18.4.78). Les larves sont présentes dans les prélèvements jusqu'au 18 juillet environ. Émergences du 27 juin au 11 juillet. Larves de la génération d'été présentes à partir du 27 juin. Émergences du 1 er au 8 août. Larves à éclosion différée à partir du 25 juillet.

Le cycle évolutif de ces 5 espèces étant le même, nous le résumons ci-dessous. La durée des générations est soumise à des fluctuations annuelles (tableau I). Nous indiquons dans ce résumé les dates limites observées pour les 3 premières espèces, numériquement les plus significatives :

- génération de larves de printemps se terminant entre le 17 juin et le 25 juillet ;

- émergence des imagos de printemps débutant entre le 9 mai et le 3 juin, et se terminant entre le 17 juin et le 25 juillet ;

- génération de larves d'été débutant entre le 30 mai et le 4 juillet et se terminant entre le 2 et le 9 septembre ;

- émergence des imagos d'été débutant entre le 8 juillet et le 25 juillet et se terminant entre le 8 août et le 26 septembre ;

- larves à éclosion différée apparaissant entre le 6 juin et le 4 juillet et disparaissant entre le 10 novembre et le 25 novembre ;

- larves de génération de printemps de l'année suivante présentes à partir du $9 \mathrm{au}$ 23 septembre.

Les espèces étudiées ici sont présentes dans la boue sous forme de larves pendant toute l'année, sans solution de continuité. Elles passent la période hivernale à l'état de larves quiescentes sous l'effet du froid; en effet, les prélèvements d'hiver ramenés au laboratoire donnent - après un temps de latence de 19 à 28 jours — des éclosions, mais en nombre plus faible que les prélèvements de printemps. Ce fait, ainsi que le temps de latence plus important pourraient suggérer une diapause hivernale touchant au moins une partie de la population.

Si le nombre de générations est constant chez ces 5 espèces, on constate par contre une très forte variation numérique d'une année à l'autre : les effectifs de C. clastrieri et de C. odibilis ont fortement augmenté de 1977 à 1978, C. musilator qui avait des effectifs presque inexistants en 1977 a nettement augmenté en nombre en 1978, C. cubitalis est resté stationnaire, et $C$. subfascipennis a fortement diminué en 1978. Ces fluctuations annuelles sont elles dûes aux facteurs climatiques, ou existeraitil une concurrence interspécifique, ou encore un prédateur spécifique ?

Fig. 1 à 5 .

Dynamique de la population de larves. Abscisses : nombre de jours passés au laboratoire jusqu'à l'éclosion. Ordonnées : dates des séries de prélèvements. Ordonnées des histogrammes selon l'échelle numérique dégressive indiquée au bas du tableau.

A gauche, émergence des adultes sur le terrain en fonction des dates (ordonnées ) ; importance numérique en abscisse (même échelle que celle des larves). 


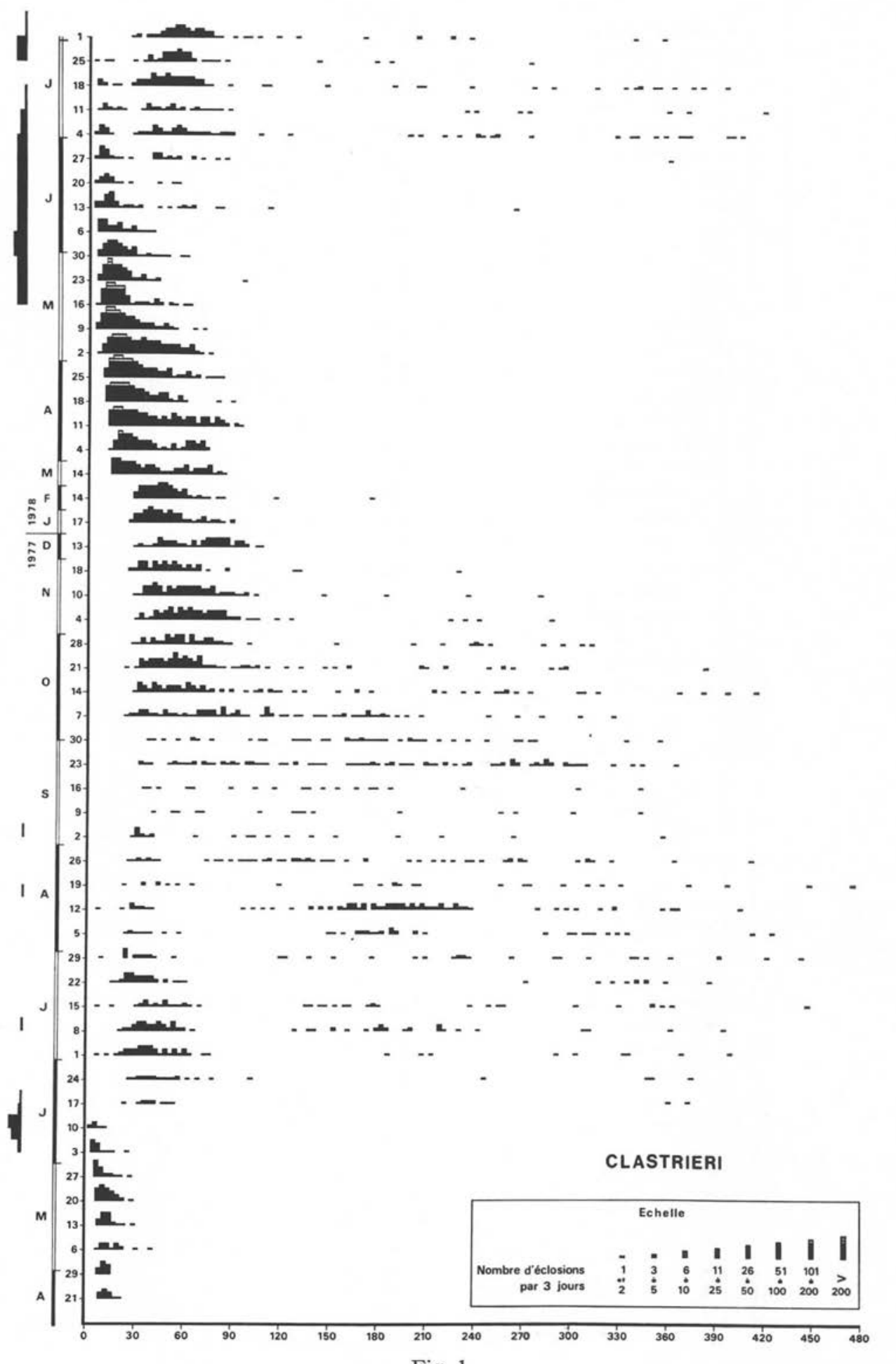

Fig. 1 


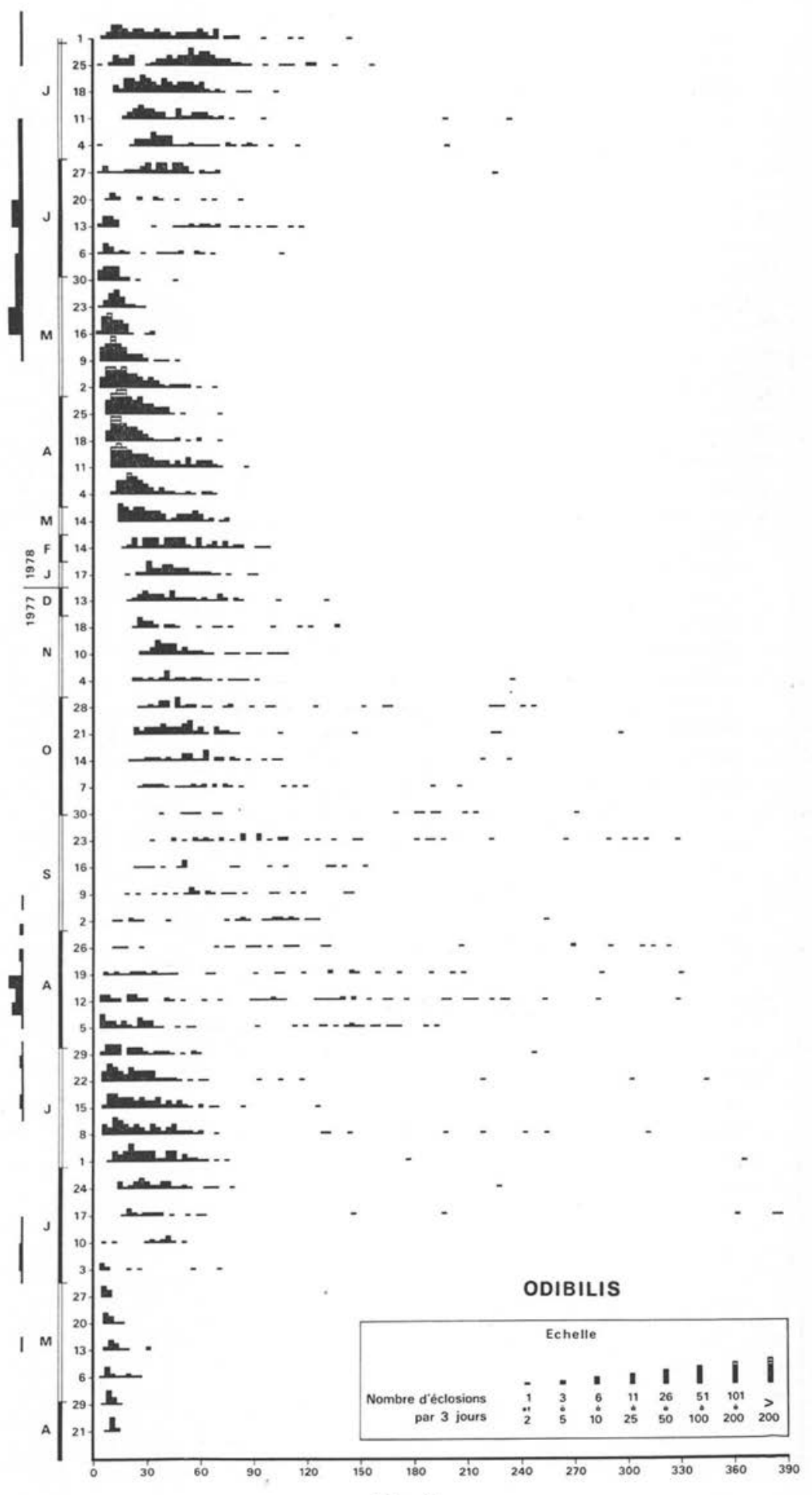

Fig. 2 


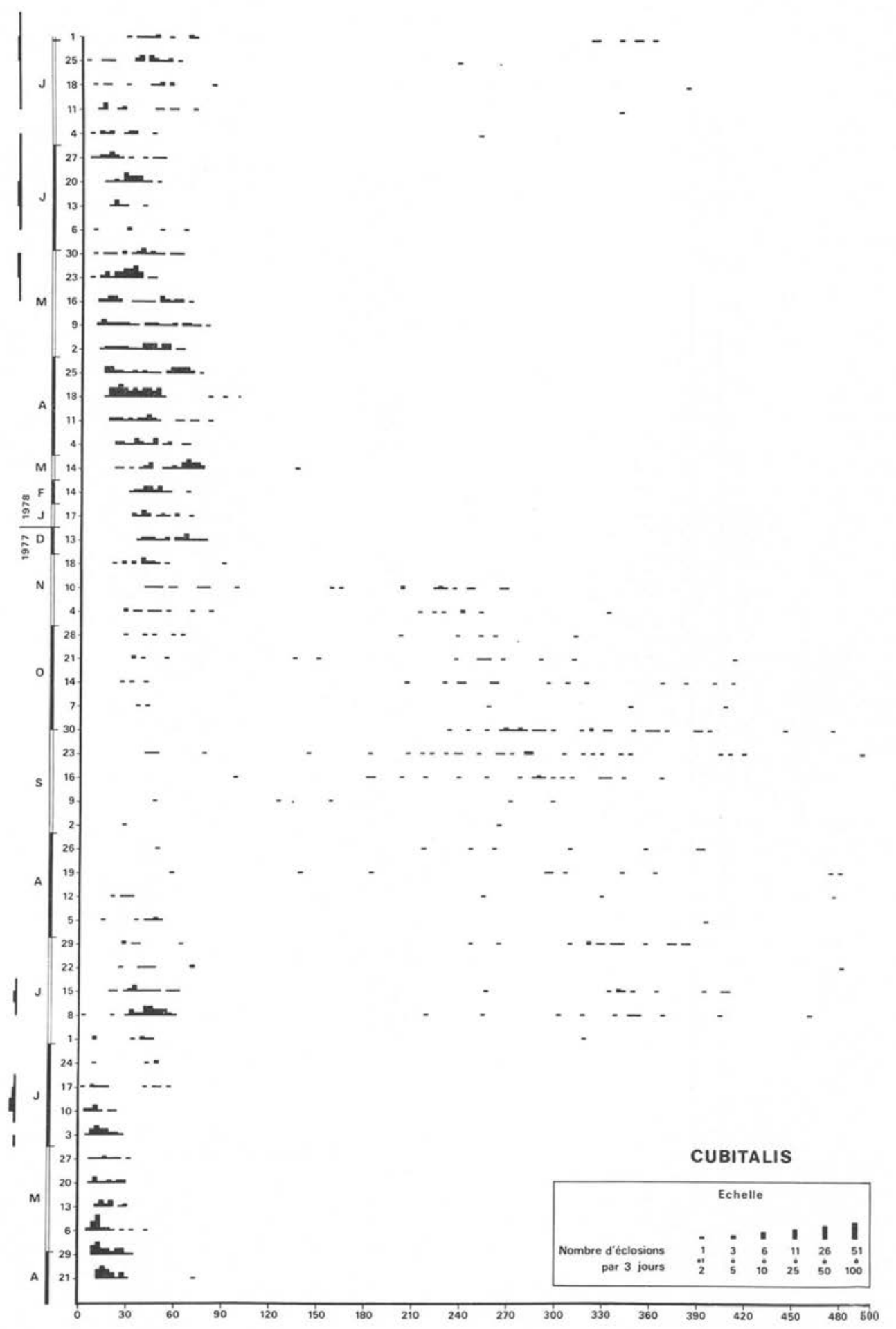

Fig. 3 


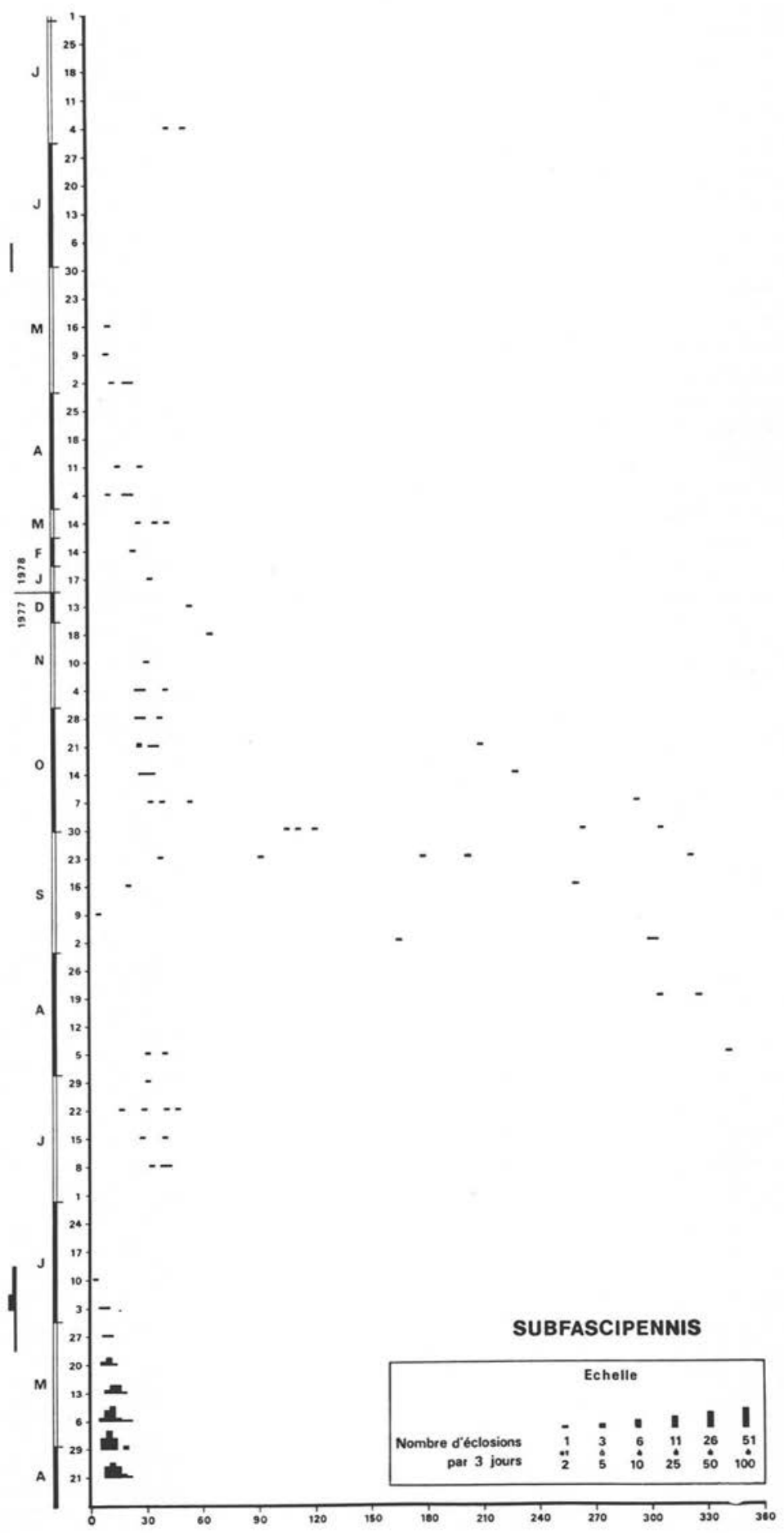

Fig. 4 


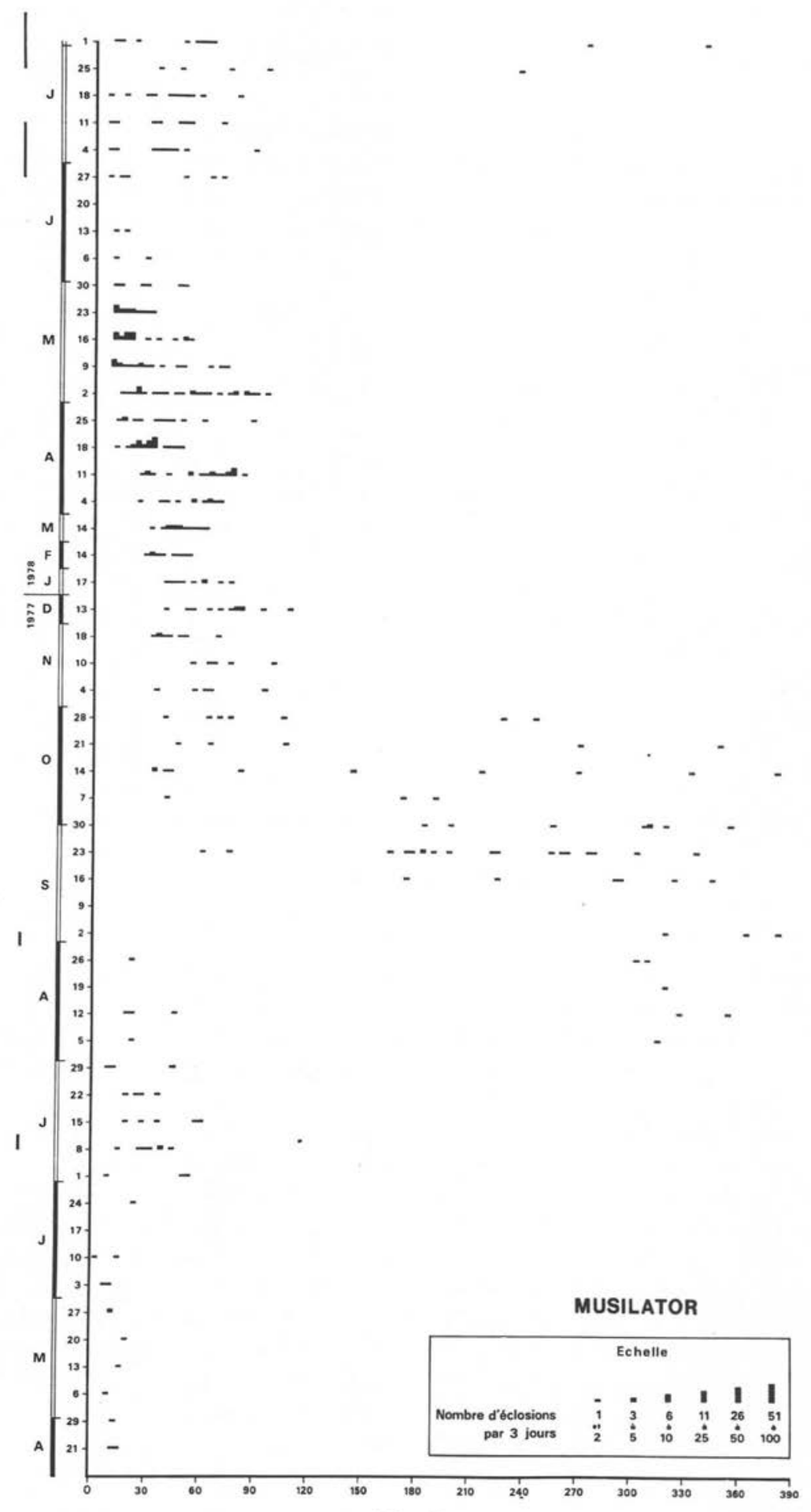

Fig. 5 
TABLEAU I : Durée des générations.

Tableau tenant compte de la présence des larves et des émergences des adultes.

\begin{tabular}{|c|c|c|c|c|c|}
\hline \multirow[b]{2}{*}{ Espèce } & \multirow[b]{2}{*}{ année } & \multicolumn{2}{|c|}{ génération de printemps } & \multicolumn{2}{|c|}{ génération d'été } \\
\hline & & début & fin & début & fin \\
\hline \multirow{2}{*}{ C. clastrieri } & 1977 & & 21 juin & 17 juin & 2 sept. \\
\hline & 1978 & 9 sept. 77 & 25 juil. 78 & 13 juin & \\
\hline \multirow{2}{*}{ C. odibilis } & 1977 & & 17 juin & 3 juin & 9 sept. \\
\hline & 1978 & 9 sept. 77 & 11 juil. 78 & 30 mai & \\
\hline \multirow{2}{*}{ C. cubitalis } & 1977 & & 8 juil. & 17 juin & 9 sept. \\
\hline & 1978 & 23 sept. 77 & 25 juil. 78 & 4 juil. & \\
\hline \multirow{2}{*}{$\begin{array}{l}\text { C. subfasci- } \\
\text { pennis }\end{array}$} & 1977 & & 14 juin & 8 juil. & 5 août \\
\hline & 1978 & 9 sept. 77 & 6 juin 78 & 一 & \\
\hline \multirow{2}{*}{ C. musilator } & 1977 & & 10 juin & 24 juin & 2 sept. \\
\hline & 1978 & 23 sept. 77 & 18 juil. 78 & 27 juin & \\
\hline
\end{tabular}

\section{B - Discussion}

\section{I - Le CYCLE ÉVOLUTIF A DEUX GÉNÉRATIONS.}

Il semblerait que beaucoup d'espèces de Culicoides vivant dans ce type de biotope humide en climat tempéré aient deux générations par an.

Albu (1971) dans des piègeages lumineux journaliers en Roumanie obtient deux pics de vol des adultes de Cératopogonidés pris dans leur ensemble : l'un en mai, l'autre en juillet.

Au Japon, Kitaoka et Morii (1963), en étudiant les populations de larves, trouvent deux générations par an pour $C$. arakawae (dans des conditions favorables, il y a une amorce de troisième génération en automne) et pour $C$. odibilis.

Hill (1947) se basant sur la récolte de nymphes près de Liverpool, trouve une seule génération par an pour $C$. odibilis et pour $C$. cubitalis. Ce résultat est surprenant pour une région qui est climatiquement proche de celle de notre étude, voir même un peu plus tempérée. Nous pensons que le comptage des nymphes confond en une courbe unimodale globale deux générations successives qui se chevauchent partiellement : ainsi l'addition des derniers individus de la génération de printemps et des premiers individus de la génération d'été donnerait la fausse impression d'une seule génération. Cette impression monocyclique est également donnée par les populations 
d'adultes étudiées par des piègeages lumineux ou à succion, pour des espèces qui sont en réalité à deux générations par an : C. arakawae (Kitaoka et Morii 1963), C. odibilis et C. cubitalis (Service 1974).

En climat plus froid, Isaev (1977) trouve dans la région de Ivanovo une seule génération pour $C$. odibilis, mais en années chaudes, il apparaît une deuxième génération qui n'est pas très importante.

2 - RENDEMENT DES ÉcLosions AU LABORATOIRE.

Nous avons montré que la technique d'élevage utilisée a un bon rendement d'éclosions ; ces dernières sont en effet plus de trois fois supérieures aux émergences d'adultes obtenues sur le terrain (Rieb et Kremer 1980). D'autre part les éclosions différées les plus tardives apparaissent encore après un séjour de 16 mois au laboratoire. On peut donc estimer que la survie des larves dans les conditions d'élevage au laboratoire est bonne, et que l'image des éclosions reflète assez bien la composition de la population larvaire présente au moment du prélèvement.

Il faut cependant remarquer que l'aspect des histogrammes est modifié sous l'effet d'une mortalité qui est liée à l'expérimentation. Cette mortalité est de deux sortes : d'une part une mortalité de laboratoire (Ml) qui affecte certains stades larvaires surtout dans les prélèvements d'automne et d'hiver, et d'autre part une mortalité de prélèvement (Mp) que l'on peut estimer à l'époque de la nymphose de printemps.

Ces modifications de l'aspect des histogrammes ne changent cependant rien à la lecture de la dynamique des populations sur les diagrammes et à la compréhension du cycle évolutif. Bien au contraire, elles révèlent des états physiologiques particuliers à des moments précis du cycle.

\section{a) La mortalité de laboratoire ( $M l)$.}

Fin septembre, tous les adultes de la génération d'été ont pondu leurs œufs. La boue contient donc un maximum d'œufs et de larves constituant la génération de printemps de l'année suivante. Ce stock d'individus reste constant pendant l'hiver et le printemps, ou diminue un peu sous l'effet d'une mortalité qui, dans la nature, paraît être très faible.

Or, le rendement des éclosions au laboratoire, des boues prélevées en septembre est très faible. Ce rendement augmente légèrement dans les prélèvements d'hiver, puis fortement dans les prélèvements du mois d'avril. Le temps de latence varie en sens inverse : il est très long en septembre ( 39 jours), il diminue lentement en hiver, puis fortement au printemps. Dans la courbe correspondante chez C. clastrieri (fig. 6 ) on a pris comme rendement maximum celui de la série de prélèvements ayant donné le plus grand nombre d'éclosions à une date précédent le début des émergences de printemps (25 avril 1978), et révélant donc le stock maximum d'individus présents dans la boue (ou proche du maximum dans le cas où on considère que la survie à $100 \%$ n'est pas entièrement atteinte).

Nous expliquons cette courbe de rendement par le fait que les larves de l'automne 
(surtout stades II et III) sont particulièrement fragiles et supportent mal les conditions du laboratoire ; la mortalité est très élevée en septembre (90 à $99 \%$ ). De janvier à avril les larves sont aux stades III et IV ; la survie augmente légèrement (mortalité de 80 à $70 \%$ ). La forte augmentation de survie en avril pourrait correspondre à un changement physiologique des larves, ce qui semble être confirmé par la diminution du temps de latence entre février et avril, à une époque où les larves n'ont pas changé de stades. Au mois de mai, les larves sont en majorité au stade IV.

Cette courbe de rendement est à considérer comme courbe de survie au laboratoire pour les prélèvements de septembre jusqu'au 16 mai (date des premières émergences). Après cette date, les émergences sur le terrain provoquent un important déficit de larves dans les prélèvements. La chute de rendement entre le 25 avril et le 16 mai est probablement imputable à la mortalité de prélèvement de stades prénymphaux.

D'autres hypothèses que celle de la mortalité des larves au laboratoire pourraient expliquer cette courbe de rendement. On pourrait imaginer que, soit des adultes hibernants (leur existence n'a jamais été démontrée dans notre région) volent aux premiers beaux jours du printemps et déposent des pontes responsables du pic du mois d'avril, soit que des œufs de l'été précédent en diapause hivernale existent dans la boue, et éclosent au printemps en formant ce pic. Mais ces hypothèses sont à rejeter, car il n'existe pas de jeunes larves dans les prélèvements de printemps. Il s'agit donc bien d'une mortalité des larves au laboratoire.

Cette mortalité pourrait s'expliquer par l'existence d'une dormance automnale et hivernale induite par le début d'abaissement de la température en automne, et nécessitant pour être levée, l'action obligatoire du froid hivernale proche de $0^{\circ} \mathrm{C}$. La mortalité serait dûe à l'absence de ce dernier. Mais les prélèvements d'automne soumis expérimentalement à des périodes variables de froid, n'ont pas vu leur rendement augmenté (Rebholtz, Kremer, Rieb 1978). Rowley (1967) constate également l'augmentation hivernale du temps de latence chez $C$. variipennis occidentalis, sans interpréter la signification du phénomène. Il obtient expérimentalement cette augmentation par action du froid sur des larves d'automne et l'attribue à l'action de ce facteur, mais ne précise pas la durée d'éclairement. Nous ne pensons pas que l'augmentation du temps de latence soit provoqué par un froid intense et prolongé, puisque nos larves de septembre n'ont pas subi une diminution de température importante et présentent déjà un temps de latence maximum. Une telle diapause hivernale existe sans doute, mais son induction éventuelle par l'abaissement de la température reste à prouver, et elle n'est pas levée par le froid hivernal.

On pourrait aussi penser à une dormance hivernale induite par la diminution d'éclairement en automne. La diapause serait levée après l'action d'un éclairement de plus en plus court en hiver, nécessitant éventuellement l'action complémentaire de l'augmentation d'éclairement au printemps. Notre recherche va s'orienter dans ce sens. Isaev (1977) dans la région d'Ivanovo, met en évidence chez $C$. odibilis une diapause hivernale dont il attribue l'induction en août et septembre à l'action de la diminution de la température et de la diminution de l'éclairement. Mais l'action du 
facteur température n'est pas clairement démontrée car elle n'est pas analysée indépendamment du facteur éclairement.

b) La mortalité de prélèvement ( $M P$ ).

Les prélèvements, même s'ils sont faits en pleine période d'émergences (mai, juin), ne donnent les premières éclosions qu'au bout de 1 à 4 jours, et le maximum d'éclosions est atteint après une dizaine de jours environ. La récolte de l'échantillon de boue, son transport et son transvasement au laboratoire, perturbent certainement les larves prêtes à nymphoser ou les nymphes. Ces stades, probablement plus fragiles, soit meurent, soit son enfouis en profondeur et voient leur développement ralenti. On peut estimer la mortalité de prélèvement chez $C$. clastrieri à 25 à $35 \%$, entre le 25 avril (rendement maximal) et le 16 mai (début des émergences) (fig 6).

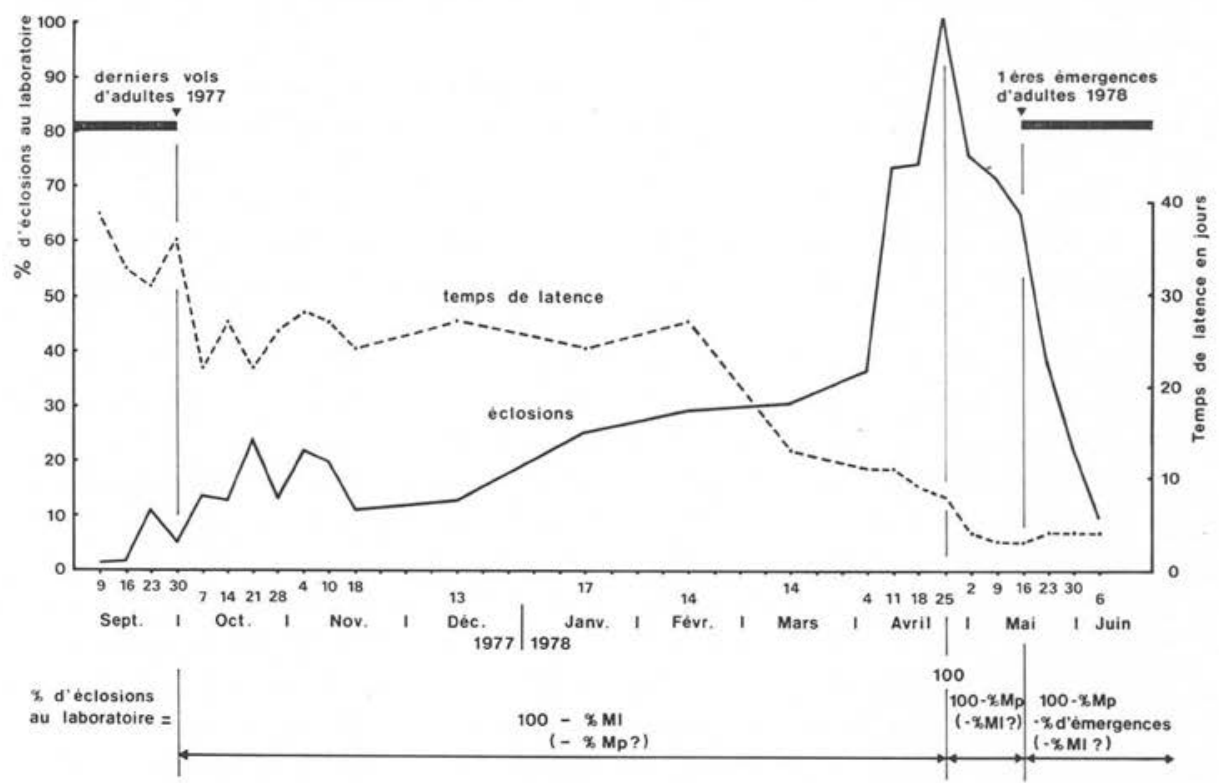

FIG. 6. - Éclosions de C. clastrieri au laboratoire : courbe de rendement des prélèvements successifs de début septembre jusqu'aux émergences de printemps. Ml = mortalité de laboratoire, $\mathrm{Mp}=$ mortalité de prélèvement.

3 - LE PHÉNOMÈNE DES ÉCLOSIONS DIFFÉRÉES : L'ESTIVO-HIBERNATION.

Une partie de la population de larves de la génération d'été subit une diapause du type estivo-hibernation (type B de Masaki 1980). Les exemplaires subissant cette diapause présentent au laboratoire une éclosion différée par rapport à ceux de la génération d'été à éclosion normale. Ces exemplaires à éclosion différée apparaissent en même temps que les premiers individus de la génération d'été. Ce sont donc des descendants de la génération de printemps. Ils sont présents dans les prélèvements pendant l'été et l'automne. Leur nombre diminue au mois de novembre et ils dispa- 
raissent fin novembre ou début décembre. Les prélèvements du printemps n'en contiennent jamais.

Il apparaît donc que les conditions de l'hiver (froid ou photopériode ?) lèvent cette diapause, puisque tous les prélèvements ayant subi l'action de l'hiver ne contiennent pas de larves à éclosion différée. Il est probable que dans la nature, les individus en estivo-hibernation voient leur diapause levée par les conditions hivernales, et éclosent au printemps suivant, après la quiescence hivernale, en même temps que la génération de printemps normale.

Au laboratoire, les prélèvements d'été et d'automne ne subissent pas les conditions de l'hiver et les individus en estivo-hibernation restent en diapause pendant un temps plus ou moins long. Cette diapause est cependant d'une durée variable d'un individu à l'autre, et elle semble être levée spontanément après un certain temps. On obtient ainsi des éclosions échelonnées dans le temps jusqu'à 16 mois après la date de prélèvement.

Dans la nature, cette levée spontanée de diapause ne peut pas avoir lieu pendant le même été. En effet, considérons l'éclosion différée la plus précoce obtenue au laboratoire : il s'agit d'un $C$. odibilis présent dans le prélèvement du 17 juin 1977 et qui éclos 5 mois après la date de prélèvement, c'est-à-dire vers la mi-novembre. A cette date, dans la nature, la diminution de température impose déjà une quiescence hivernale empêchant toute éclosion. L'exemplaire éclora dans la nature, au printemps suivant.

Dans son étude sur la diapause de $C$. odibilis en Russie, Isaev (1977) conclut à l'existence d'un seul type de diapause, qui est celle de l'hiver. Mais nous pensons, à la vue de ses résultats chiffrés que - du fait de l'absence d'une génération d'été la diapause hivernale et l'estivo-hibernation se superposent. La lecture de ses tableaux nous permet de conclure qu'il existe également une estivo-hibernation en climat continental russe dans la région d'Ivanovo.

Nous interprétons la signification de l'estivo-hibernation de la manière suivante :

En notre région, la génération d'été est parfois numériquement plus faible que la génération de printemps. Ainsi, lors d'une année à belle saison courte, la génération de printemps aura, dans tous les cas, le temps de se développer, mais la génération d'été risque de rencontrer précocément des conditions météorologiques défavorables, d'être réduite et de mettre la pérennité de l'espèce en danger. Nous savons également qu'en climat plus continental, la génération d'été est très faible et souvent inexistante. La génération d'été est donc aléatoire.

L'estivo-hibernation correspondrait à un mécanisme de sécurité assurant la survie de l'espèce en cas de défaillance de la génération d'été. En effet, les individus de la génération d'été de l'année n qui entrent en diapause, sont mis en réserve et à l'abri de l'évolution hasardeuse de cette génération d'été. Ils n'éclosent qu'au printemps de l'année $\mathrm{n}+1$ en même temps que la génération de printemps $\mathrm{n}+1$.

L'estivo-hibernation pourrait aussi - dans le cas d'une petite population isolée géographiquement - assurer une régénération du patrimoine héréditaire par croisement entre une génération $n$ et une génération $n+1$, et éviter ainsi la consanguinité. 


\section{BIBLIOGRAPHIE}

Albu P. : On the Chironomids (Diptera, Chironomidae) captured in a light-trap in Sinaia (Rumania). Limnologica (Berlin), I971, 8, I57-I 72.

Hill M. A. : The life-cycle and habits of Culicoides impunctatus Goetghebuer and Culicoides obsoletus Meigen, together with some observations on the life-cycle of Culicoides odibilis Austen, Culicoides pallidicornis Kieffer, Culicoides cubitalis Edwards and Culicoides chiopterus Meigen. Ann. Trop. Med. Parasit., I947, 4I, 55-II5.

IsAEV V. A. : The influence of the environmental factors on the formation and overcoming of diapausa in Culicoides odibilis Austen larvae (Diptera, Ceratopogonidae). Med. Parazit. Moskva, 1977, 46, 209-214.

KITAOKA S., MORII T. : Observations on the breeding habitats of some biting midges and seasonal population dynamics in the life cycle of Culicoides arakawae in Tokyo and its vicinity. Nat. Inst. Anim. Hlth. Quart., 1963, 3, 198-208.

MAZAKI S. : Summer diapause. Ann. Rev. Entomol., I980, 25 , I-25.

Rebholtz C., KREMER M., Rieb J.-P. : Rôle de la lumière et de la température sur le développement de C. riethi et C. nubeculosus en élevage. Ann. Parasitol. Hum. Comp., I979, 54, 257-258.

RIEB J.-P., KREMER M. : Écologie des Cératopogonidés de la plaine d'Alsace. II. Répartition spatiale des larves de Culicoides d'un gite fluviatile. Ann. Parasitol. Hum. Comp., 1980, 55, $59 \mathrm{I}-603$.

ROWLEY W. A. : Observations on larval habitats and the winter bionomics of some common species of Culicoides (Diptera : Ceratopogonidae) in the Central Columbia Basin. Mosq. News, $1967,27,499-505$.

Service M. W. : Further results of catches of Culicoides (Diptera : Ceratopogonidae) and mosquitoes from suction traps. J. Med. Ent., I974, II, 471-479. 